\title{
LA MOTIVACIÓN DE LOS ESTUDIANTES UNIVERSITARIOS PARA REALIZAR ACTIVIDAD FÍSICA
}

\author{
Miguel Ángel Durán-Vinagre \\ Dpto. Psicología y Antropología \\ Universidad de Extremadura. Badajoz (España) \\ mduranv@unex.es \\ Susana Sánchez Herrera \\ Dpto. Psicología y Antropología \\ Universidad de Extremadura. Badajoz (España) \\ ssanchez@unex.es \\ Sebastián Feu \\ Dpto. Expresión Plástica, Musical y Corporal \\ Universidad de Extremadura. Badajoz (España) \\ sfeu@unex.es
}

Recepción Artículo: 16 mayo 2021 Admisión Evaluación: 16 mayo 2021 Informe Evaluador 1: 27 mayo 2021

Informe Evaluador 2: 02 junio 2021

Aprobación Publicación: 03 junio 2021

\section{RESUMEN}

La actividad deportiva tiene un papel importante e insustituible en la adolescencia, ya que su práctica regular no sólo protege a las personas contra las enfermedades no transmisibles, sino que también contribuye a una mejor calidad de vida. El objetivo de este estudio fue analizar el tipo de regulación de las motivaciones y los motivos para la realización de actividad física que presentan los estudiantes en función de la variable sexo. Participaron un total de 170 estudiantes universitarios del primer curso del Grado de Maestro, el 35.3\% hombres $(n=60)$ y el $64.7 \%$ mujeres $(n=110)$, con una media de edad de 20.02 \pm 2.55 . Respondieron al Cuestionario de Regulación de la Conducta en el Ejercicio (BREQ-3) y al Cuestionario de Medida de los motivos de Actividad Física Revisada (MPAM-R). Los resultados revelan diferencias estadísticamente significativas en las variables regulación intrínseca $(U=1418.5 ; p<.05)$, integrada $(U=1520.5 ; p<.05)$ e identificada $(U=2157.5 ; p<.05)$. Del mismo modo, se obtuvo diferencias estadísticamente significativas en los motivos para la realización de actividades físicas en las variables de disfrute $(U=1441.5 ; p<.05)$, social $(U=1917.5 ; p<.05)$, fitness/salud $(U=2533$; $p<.05)$ y competencia $(U=1622.5 ; p<.05)$. Con estos datos se puede concluir que los motivos para participar en la actividad física presentan una influencia en los estudiantes universitarios de diferente manera, ya que los más valorados por los participantes son los que tienen una asociación intrínseca y más autodeterminada hacia la propia práctica deportiva.

Palabras clave: actividad física; hábitos saludables; motivación; adolescentes 


\section{LA MOTIVACIÓN DE LOS ESTUDIANTES UNIVERSITARIOS PARA REALIZAR ACTIVIDAD FÍSICA}

\section{ABSTRACT}

The motivation of university students to engage in physical activity. Sports activity plays an important and irreplaceable role in adolescence, since its regular practice not only protects people against noncommunicable diseases, but also contributes to a better quality of life. The objective of this study was to analyze the type of regulation of motivations and motives for physical activity that students present according to the gender variable. A total of 170 university students from the first year of the Master's Degree participated, 35.3\% men (n $=60)$ and $64.7 \%$ women $(n=110)$, with a mean age of 20.02

\pm 2.55 . They responded to the Exercise Behavior Regulation Questionnaire (BREQ-3) and the Revised Physical Activity Measurement Questionnaire (MPAM-R). The results reveal statistically significant differences in the variables intrinsic regulation $(U=1418.5 ; p<.05)$, integrated $(U=1520.5 ; p<.05)$ and identified $(U=2157.5$; $p<.05)$. Similarly, statistically significant differences were obtained in the reasons for carrying out physical activities in the variables of enjoyment $(U=1441.5 ; p<.05)$, social $(U=1917.5 ; p<.05)$, fitness / health $(U=2533$; $p<.05)$ and competence $(U=1622.5 ; p<.05)$. With these data, it can be concluded that the reasons for participating in physical activity have an influence on university students in different ways, since those most valued by the participants are those with an intrinsic and more self-determined association towards their own sports practice.

Keywords: physical activity; healthy habits; motivation; teenagers

\section{INTRODUCCIÓN}

La adolescencia constituye una etapa de gran importancia en la creación de hábitos de salud, pues representa el periodo de transición hacia la vida adulta en el que se configuran estilos de vida (Güemes-Hidalgo, Ceñal e Hidalgo, 2017). En este sentido, la actividad física de calidad estimula el desarrollo físico, cognitivo y social de los adolescentes y además favorece la creación de hábitos de práctica física saludables (Duda y Ntounamis, 2003).

En los últimos años, las investigaciones sobre la actividad física y la salud han aumentado considerablemente. Estos estudios muestran que realizar ejercicio físico de forma regular y continuo está directamente relacionado con la mejora de la salud y el aumento de la esperanza de vida (Cruz-Santos, González-Rivera y RosarioRodríguez, 2019; Lavie et al., 2019; Rippe, 2019). Si bien, aunque existen dichas evidencias científicas que demuestran esta relación, la conducta de inactividad física y el sedentarismo se ha convertido en uno de los principales inconvenientes de la sociedad actual en el plano nacional e internacional (García, 2019; Pérez, 2014), hallando trabajos que concluyen que la proporción de jóvenes que realizan actividades deportivas es muy baja 0 escasa (Chuliá et al., 2005; Durán-Vinagre, Sánchez y Feu, 2019; Pérez, Requena y Zubiaur, 2005). Estos datos se deben mayormente a que las personas priorizan otras actividades diarias y de ocio sin evaluar los beneficios y efectos positivos de la actividad física a nivel físico y psíquico (Chuliá et al., 2005; Molina, Castillo, Pablos, 2007).

Para dar una explicación a estos datos, la actividad física se ha asociado con los procesos cognitivos (Doherty y Forés, 2019; Reloba, Chirosa y Reigal, 2016), los procesos emocionales (Hagenauer, Gläser-Zikuda y Moschner, 2018), y principalmente con la motivación (Franco, Coteron y Gómez, 2017; Chacón et al., 2017; Vaquero-Solís et al., 2019). Si consideramos este último constructo, la motivación presenta un papel vital en la práctica y la promoción del ejercicio físico en el ser humano, ya que éstos se comportan y actúan de acuerdo con unos determinados aspectos motivacionales a la hora de adquirir y alcanzar sus propias metas (Almagro, Navarro, Paramio y Sáenz-López, 2015).

A este respecto, la motivación determina la dirección y la intensidad de la conducta a la hora de participar en actividades físico-deportivas, entendiéndose por dirección aquellas posibles metas que una persona manifiesta al sentirse atraído por ella y por intensidad a la cantidad de empeño y esfuerzo que una persona emplea para alcanzar determinadas metas (Escartí y Brustad, 2002). Tanto la intensidad como la dirección que adoptan los motivos de práctica son relevantes para la continuidad en una actividad física y/o deportiva (Cervelló, Escartí y Guzmán, 2007). 
Profundizando aún más sobre la motivación, ésta presenta una estrecha asociación con los motivos de la práctica deportiva (Castañeda et al., 2018). Los motivos que suscitan a los adolescentes a practicar actividad física son similares a los que presenta la población general (Moral, Martínez y Grao, 2013). A este respecto, la mayoría de las investigaciones realizadas con jóvenes universitarios muestran que éstos a la hora de realizar actividad física manifiestan motivos relacionados con actividades placenteras y que conlleven una implicación social (Castañeda-Vázquez et al., 2014; Granero-Gallegos et al., 2011), aunque depende del perfil y características de cada persona (Chacón et al., 2018). Del mismo modo, es conveniente resaltar la motivación de los estudiantes hacia la actividad física por una mejora o mantenimiento de la salud (Corbí, Palmero-Cámara y Jiménez- Palmero, 2019), la cual se mantiene y se prolonga a lo largo del tiempo una vez finalizados los estudios universitarios (Almorza, Jiménez y Molero, 2011).

En cualquier caso, es fundamental seguir estudiando y ahondando la motivación que manifiestan los futuros docentes (ahora estudiantes universitarios de primer curso) en relación con la actividad física y los motivos que le genera la realización de su práctica, de manera que sea posible adecuar y adaptar las características de la práctica deportiva para dar respuesta a las demandas de este grupo poblacional, además de planificar y diseñar actividades acordes a sus propios intereses.

\section{OBJETIVO DE INVESTIGACIÓN}

El objetivo del trabajo fue analizar el tipo de regulación de las motivaciones y los motivos para la realización de actividad física que presentan los estudiantes en función de la variable sexo.

\section{MATERIALES Y MÉTODOS}

\section{Participantes}

La muestra estuvo constituida por 170 estudiantes universitarios del primer curso del Grado de Maestro, el $35.3 \%$ hombres $(n=60)$ y el $64.7 \%$ mujeres $(n=110)$, con una media de edad de $20.02 \pm 2.55$.

\section{Instrumentos}

Para el análisis del tipo de motivación en relación con la AF, se utilizó la escala Behavioral Regulation in Exercise Questionnaire-3, versión BREQ-3, validada al español (González-Cutre, Sicilia y Fernández, 2010). El cuestionario BREQ-3 está formado por 23 ítems agrupados en seis factores. La escala de respuesta es de tipo Likert puntuando desde 0 (nada verdadero) hasta 4 (totalmente verdadero). Los factores son: motivación Intrínseca (=.94), Integrada (=.96), Identificada (=.83), Introyectada (=.73), Externa (=.81) y Desmotivación ( =.83). Los valores del Alfa de Cronbach fueron adecuados ( >.70) (Nunnally y Bernstein, 1994).

Para los motivos de práctica para el ejercicio físico se empleó el cuestionario Motives for Physical Activity Measure-Revised (MPAM-R) de Ryan et al. (1997), versión validada al español (Moreno-Murcia, Cervelló y Martínez, 2007). Este instrumento está constituido por cinco factores a través de 30 ítems en una escala Likert de 1 (totalmente en desacuerdo) al 7 (totalmente de acuerdo). Los factores para este estudio son: Fitness (=.91), Apariencia (=.91), Disfrute (=.95), Social (=.88) y el factor Competencia (=.94). La fiabilidad de las escalas utilizadas fue óptima ( >.85) (Nunnally y Bernstein, 1994).

\section{Procedimiento}

Se solicitó el consentimiento informado a la Facultad de Educación y a todos los participantes que cumplimentaron el cuestionario. Se informó que su participación era voluntaria y anónima, respetándose la Ley orgánica 15/ 1999, del 13 de diciembre de protección de datos. Todos los participantes fueron tratados de acuerdo con los principios éticos y los códigos de conducta de la Asociación Americana de Psicología (APA, 2010). Los cuestionarios se completaron en 12-15 minutos. Durante la administración del instrumento, al menos un investigador estaba presente en el aula, sin que ninguno de los participantes informara dificultades a la hora de contestar. 


\section{LA MOTIVACIÓN DE LOS ESTUDIANTES UNIVERSITARIOS PARA REALIZAR ACTIVIDAD FÍSICA}

\section{Análisis estadístico}

De acuerdo con el objetivo de este estudio y en función de la naturaleza de las variables analizadas se realizó un análisis de datos descriptivo e inferencial, aplicando la prueba de la U de Mann-Whitney junto con los rangos promedios de los hombres y de las mujeres. El nivel de confianza que se consideró fue de un 95\%. Para el tratamiento estadístico de los datos se utilizó el programa estadístico para las ciencias sociales (Statistical Package for the Social Sciences, SPSS) versión 20 (Nie, Hull y Bent, 2011).

\section{RESULTADOS}

En la regulación de las motivaciones se han encontrado diferencias estadísticamente significativas en las variables regulación intrínseca $(U=1418.5 ; p<.05)$, integrada $(U=1520.5 ; p<.05)$ e identificada $(U=2157.5 ; p<.05)$. Las medias de los hombres son significativamente mayores que las de las mujeres en las variables más orientadas hacia la regulación interna para la práctica de actividad físico-deportiva. Ambos sexos, otorgan puntuaciones bajas para la desmotivación (hombres .37 y las mujeres .60) y la regulación externa (hombres .24 y las mujeres .36), no existiendo, por tanto, diferencias significativas en estos tipos de motivación y regulación (Tabla 1).

Se encontraron diferencias estadísticamente significativas en los motivos para la realización de actividades físicas en las variables de disfrute $(U=1441.5 ; p<.05)$, social $(U=1917.5 ; p<.05)$, fitness/salud ( $U=2533 ; p<.05)$ y competencia $(U=1622.5 ; p<.05)$. Los rangos promedios son significativamente mayores en hombres que en mujeres en cuanto a la diversión, la socialización, la mejora por la salud y el propio gusto hacia los desafíos. Sin embargo, no existían diferencias significativas en función de la variable apariencia.

Tabla 1. Análisis descriptivo e inferencial de las variables del estudio en función del sexo.

\begin{tabular}{|c|c|c|c|c|c|c|c|c|c|}
\hline & \multirow{2}{*}{ Variables } & \multicolumn{2}{|c|}{ Hombres } & \multicolumn{2}{|c|}{ Mujeres } & \multirow{2}{*}{$\begin{array}{l}\text { U Mann } \\
\text { Whitney }\end{array}$} & \multirow{2}{*}{$p$} & \multirow{2}{*}{$\begin{array}{c}\text { Rango } \\
\text { promedio } \\
\text { hombres }\end{array}$} & \multirow{2}{*}{$\begin{array}{c}\text { Rango } \\
\text { promedio } \\
\text { mujeres }\end{array}$} \\
\hline & & M & D.t & $\mathbf{M}$ & D.t & & & & \\
\hline \multirow{6}{*}{ BREQ-3 } & Intrínseca & 3.19 & .968 & 2.04 & 1.158 & 1418.5 & $*$ & 116.86 & 68.40 \\
\hline & Integrada & 2.83 & 1.161 & 1.65 & 1.172 & 1520.5 & $*$ & 115.16 & 69.32 \\
\hline & Identificada & 3.14 & .896 & 2.55 & 1.046 & 2157.5 & $*$ & 104.54 & 75.11 \\
\hline & Introyectada & 1.04 & .818 & .94 & .800 & 3027.5 & - & 90.04 & 83.02 \\
\hline & Externa & .24 & .524 & .36 & .650 & 3035.5 & - & 81.09 & 87.90 \\
\hline & Desmotivación & .37 & .592 & .60 & .918 & 2991.5 & - & 80.36 & 88.30 \\
\hline \multirow{5}{*}{ MPAM-R } & Disfrute & 5.67 & .982 & 4.21 & 1.599 & 1441.5 & $*$ & 116.48 & 68.60 \\
\hline & Apariencia & 4.68 & 1.394 & 4.59 & 1.569 & 3192 & - & 87.30 & 84.52 \\
\hline & Social & 4.53 & .929 & 3.56 & 1.348 & 1917.5 & $*$ & 108.54 & 72.93 \\
\hline & Fitness & 5.82 & .888 & 5.22 & 1.432 & 2533 & $*$ & 98.28 & 78.53 \\
\hline & Competencia & 5.48 & 1.042 & 4.20 & 1.532 & 1622 & $*$ & 113.47 & 70.25 \\
\hline
\end{tabular}

*La diferencia de medias es significativa al nivel .05 $(p<.05)$.

Se encontró una asociación alta y significativa entre las actividades realizadas y el sexo ( $X^{2}=44.779 ; g$ l= 6; $p=.000 ; V_{c}=.513 ; p=.000$ ). Los resultados indicaron que las actividades de deporte colectivo eran más significativas en hombres ( $R T C=6.3$ ), mientras que las mujeres preferían significativamente andar $(R T C=2)$. Igualmente, los datos demuestran que las mujeres presentan más casos de los esperados cuando hablamos del valor de actividad físico-deportiva que refiere a la casilla en blanco o no se especifica $(R T C=2,5)$ (Tabla 2). 
Tabla 2. Tabla de contingencia de actividades deportivas que realizan agrupadas por sexo.

\begin{tabular}{|c|c|c|c|c|}
\hline \multirow{2}{*}{\multicolumn{2}{|c|}{ Actividades deportivas }} & \multicolumn{2}{|c|}{ Sexo } & \multirow{2}{*}{ Total } \\
\hline & & Hombre & Mujer & \\
\hline \multirow{4}{*}{$\begin{array}{c}\text { En blanco o no } \\
\text { especifica }\end{array}$} & Recuento & 15 & 49 & 64 \\
\hline & Frecuencia esperada & 22.6 & 41.4 & 64 \\
\hline & $\%$ del total & $8.8 \%$ & $28.8 \%$ & $37.6 \%$ \\
\hline & RTC & $-2,5$ & 2.5 & \\
\hline \multirow{4}{*}{ Andar } & Recuento & 1 & 11 & 12 \\
\hline & Frecuencia esperada & 4.2 & 7.8 & 12 \\
\hline & $\%$ del total & $0.6 \%$ & $6.5 \%$ & $7.1 \%$ \\
\hline & RTC & -2.0 & 2.0 & \\
\hline \multirow{4}{*}{ Correr } & Recuento & 10 & 22 & 32 \\
\hline & Frecuencia esperada & 11.3 & 20.7 & 32 \\
\hline & $\%$ del total & $5.9 \%$ & $12.9 \%$ & $18.8 \%$ \\
\hline & RTC & -.5 & .5 & \\
\hline \multirow{4}{*}{ Deporte colectivo } & Recuento & 21 & 1 & 22 \\
\hline & Frecuencia esperada & 7.8 & 14.2 & 22 \\
\hline & $\%$ del total & $12.4 \%$ & $0.6 \%$ & $12.9 \%$ \\
\hline & $R T C$ & 6,3 & -6.3 & \\
\hline \multirow{4}{*}{ Deporte individual } & Recuento & 4 & 4 & 8 \\
\hline & Frecuencia esperada & 2.8 & 5.2 & 8 \\
\hline & $\%$ del total & $2.4 \%$ & $2.4 \%$ & $4.7 \%$ \\
\hline & $R T C$ & .9 & -.9 & \\
\hline \multirow{4}{*}{$\begin{array}{l}\text { Deporte con } \\
\text { implemento }\end{array}$} & Recuento & 1 & 1 & 2 \\
\hline & Frecuencia esperada & .7 & 1,3 & 2 \\
\hline & $\%$ del total & $0.6 \%$ & $0.6 \%$ & $1.2 \%$ \\
\hline & RTC & .4 & -.4 & \\
\hline \multirow{4}{*}{$\begin{array}{l}\text { Actividades Centro } \\
\text { Fitness }\end{array}$} & Recuento & 8 & 22 & 30 \\
\hline & Frecuencia esperada & 10.6 & 19.4 & 30 \\
\hline & $\%$ del total & $4.7 \%$ & $12.9 \%$ & $17.6 \%$ \\
\hline & $R T C$ & -1.1 & 1.1 & \\
\hline \multirow{2}{*}{ TOTAL } & Recuento & 60 & 110 & 170 \\
\hline & $\%$ del total & $35.3 \%$ & $64.7 \%$ & $100.0 \%$ \\
\hline
\end{tabular}

\section{DISCUSIÓN}

Considerando el objetivo de este estudio que fue analizar el tipo de regulación de las motivaciones y los motivos para la realización de actividad física que presentan los estudiantes en función de la variable sexo podemos partir con la afirmación realizada por Vidarte et al. (2011) quienes entienden que la actividad física es una estrategia eficaz en la promoción de la salud. Para incentivar y sembrar su práctica, las universidades deben centrarse en la conducta y los motivos que presentan los estudiantes para su realización, ya que la intervención debe estar dirigida a suscitar un cambio a través de la satisfacción de las necesidades de éstos.

Los resultados de la presente investigación indicaron que los estudiantes universitarios puntuaban más alto en los factores de la regulación intrínseca (3.19), identificada (3.14) e integrada (2.83) y más bajo en la regulación externa (.24) y la desmotivación (.37), teniendo como referencia la puntuación máxima siendo el valor 5 . Por lo contrario, las mujeres otorgaron valores medios inferiores a los hombres en la regulación intrínseca (2.04), identificada (2.55) e integrada (1.65) y más altos en la regulación externa (.36) y la desmotivación (.60). Estos datos tienen cierta coherencia, ya que el hecho de realizar actividades físico-deportivas de manera voluntaria se asocia a una motivación autodeterminada alta, es decir, con una alta regulación identificada, intrínseca e integrada y una 


\section{LA MOTIVACIÓN DE LOS ESTUDIANTES UNIVERSITARIOS PARA REALIZAR ACTIVIDAD FÍSICA}

baja regulación externa o desmotivación (Muyor et al., 2009). En consonancia, Kim y Gill (1997) y Amorose y Horn (2000) reflejan que los hombres presentan una mayor motivación intrínseca que las mujeres. Estos resultados corroboran Ios encontrados en otros estudios (Granero-Gallegos et al., 2012; Martínez et al., 2015; Moreno, González- Cutre y Ruiz, 2009).

Por lo tanto, en este trabajo se han encontrado diferencias estadísticamente significativas en cuanto a la regulación de la motivación intrínseca, integrada e identificada en relación con el sexo. Estos resultados no se ajustan a los obtenidos en otros estudios tales como el de Muyor et al. (2009) en el que no manifestaron diferencias significativas en la regulación de las motivaciones en función del sexo, a excepción de la desmotivación que fue más destacable en hombres que en mujeres. Zamarripa et al. (2011) señala que la desmotivación es un factor psicológico que puede originar la inactividad física.

Respecto a los motivos de la práctica física, Koivula (1999) en su investigación obtuvo que los hombres presentaban mayores puntuaciones en el factor de competencia que las mujeres, quien apuntó que ellos muestran una actitud más competitiva como el principal motivo de la participación deportiva. Estos datos, también se manifiestan en este estudio, donde se observó que hay diferencias estadísticamente significativas en cuanto al sexo, siendo los hombres quienes puntuaban más alto que las mujeres.

En esta línea, encontramos a Fernández et al. (2015) en la que estudian a una muestra de 455 estudiantes, en donde sus principales motivos radican en el fitness (6.14), el disfrute (5.89) y la competencia (5.71) revelaron diferencias significativas en cuanto al disfrute, la apariencia, lo social y la competencia, de manera que los hombres presentaban un mayor promedio que en las mujeres, mientras que ellas puntuaban más alto en la apariencia. Estos hallazgos son consistentes con nuestros datos ya que los hombres muestran un mayor promedio de puntuaciones en todos los motivos respecto a las mujeres.

Considerando el estudio de Castañeda y Campos-Mesa (2012), sus resultados desvelaron que los principales motivos por los estudiantes universitarios de la Facultad de Ciencias de la Educación de Sevilla estaban asociados a la mejora de la salud y la estética, principalmente, además del disfrute y las relaciones sociales. Datos que se asocian a los obtenidos en este estudio, en donde se encuentran diferencias estadísticamente significativas en todos los motivos (disfrute, social, fitness y competencia) exceptuando en la apariencia. También Liu et al. (2017) obtienen diferencias significativas en cuanto al disfrute y la competencia, siendo predictores positivos en el que se asocia a la motivación intrínseca en la medida en que dichos motivos se centraban en los aspectos inherentes de la actividad física, es decir, el valor de interés, el deseo de desafíos o el ejercicio de habilidades.

\section{CONCLUSIONES}

Para mejorar los niveles de actividad físico-deportiva en los estudiantes universitarios sería de gran utilidad compartir y revelar los resultados obtenidos en el presente estudio para que éstos valoren la importancia que tiene realizar actividad física de manera continua y moderada, ya que los datos hallados reflejan unos niveles bajos de actividades físicas en la población objeto de estudio. En este sentido se aconseja seguir y mantener las recomendaciones planteadas por la Organización Mundial de la Salud o American College of Sports Medicine para hacer actividad física. Del mismo modo, también es necesario promover políticas adecuadas para la promoción de la actividad física, y desarrollar planes de intervención para establecer hábitos saludables en el que la influencia de la regulación de las motivaciones y los motivos de práctica físico deportiva del alumnado universitario se vea mejorado. Así pues, estos programas deben enfocarse en atender las necesidades de los estudiantes en función del sexo, ya que los gustos por la práctica son diferentes en sendos casos.

Por otra parte, es conveniente proponer actividades que permitan modificar la conducta de los participantes, de manera que los participantes que muestran una motivación más extrínseca puedan ser redirigidos a conductas más autodeterminadas, es decir, enfocar y proyectar la motivación de la actividad física hacia un locus de causalidad más interno. Esta situación dará la posibilidad de mejorar los procedimientos de autorregulación inherentes a la práctica, el disfrute e incluso a la satisfacción por la actividad física. 
Finalmente, debemos brindar actividades que se conviertan en motivos orientados hacia aquellas prácticas que generen vínculos de relaciones sociales, la diversión y la mejora de la salud, evitando propiciar ambientes enfocados hacia la apariencia física o la competencia, pues estas causas se vinculan a motivaciones que ofrecen recompensas externas o reconocimientos públicos de cánones de belleza corporal.

\section{AGRADECIMIENTOS}

Agradecemos la financiación de las ayudas a grupos de la Junta de Extremadura (GR18132) (GR18170) y FEDER, una manera de hacer Europa. También agradecemos la colaboración del Plan Propio de Iniciación a la Investigación, Desarrollo Tecnológico e Innovación de la Universidad de Extremadura 2020.

\section{REFERENCIAS BIBLIOGRÁFICAS}

Almagro, B. J., Navarro, I., Paramio, G., y Sáenz-López, P. (2015). Consecuencias de la motivación en las clases de Educación Física. Revista Digital de Educación Física, 6(34), 26-41.

Almorza, D., Jiménez, J.A., y Molero, J. (2011). Estudio diagnóstico del deporte universitario español. Madrid: Consejo Superior de Deportes.

American Psychological Association (2010). 2010 Amendments to the 2002 "Ethical principles of psychologists and code of conduct". American Psychologist, 65(5), 493.

Amorose, A. J., y Horn, T. S. (2000). Intrinsic Motivation: relationships with collegiate athletes gerder, scholaship status, and perceptions of their coaches behavior. Journal of Sport \& Exercise Psychology, 22, 63-84.

Castañeda, C., y Campos-Mesa, M. C. (2012). Motivación de los estudiantes de la Facultad de Ciencias de la Educación (Universidad de Sevilla) hacia la práctica de actividad físico-deportiva. Retos. Nuevas tendencias en Educación Física, Deporte y Recreación, 22, 57-61.

Castañeda, C., Zagalaz, M. L., Arufe, V., y Campos-Mesa, M. C. (2018). Motivos hacia la práctica de actividad física de los estudiantes universitarios sevillanos. Revista Iberoamericana de Psicología del Ejercicio y el Deporte, 13(1), 79-89.

Castañeda-Vázquez, C., Zagalaz-Sánchez, M.L., Chacón-Borrego, F., Cachón-Zagalaz, J., y Romero-Granados, S. (2014). Características de la práctica deportiva en función del género. Estudiantes de la Facultad de Ciencias de la Educación: Universidad de Sevilla. Retos Nuevas tendencias en educación física, deporte y recreación, 25, 63-67.

Cervelló, E., Escartí, A., y Guzmán, J. F. (2007). Youth sport dropout from the achievement goal Theory. Psicothema, 65-71.

Chacón, R., Chacón, F., Zurita, F., Cachón, J., Zagalaz, M.L., y Chinchilla, J.J. (2018) Characterization of motivation and type of physical sport practice in adults through COMPASS proiles. Journal of Human Sport and Exercise, 13(1), 161- 173.

Chuliá, M., Ferrer, E., Lizama, N., Martin, S., y Monrabal, C. (2005). El sedentarismo en los jóvenes universitarios. Educare 21, 17, 125-129.

Corbí, M., Palmero-Cámara, C., y Jiménez-Palmero, A. (2019). Diferencias en los motivos hacia la actividad física de los universitarios según nivel de actividad y su relación con la satisfacción del servicio deportivo universitario. Retos Nuevas tendencias en educación física, deporte y recreación, 35, 191-195.

Cruz-Santos, A., González-Rivera, J. A, Rosario-Rodríguez, A. (2019). Attitudes towards physical exercise and sports: A psychometric study in college students. Revista Evaluar, 19(1), 58-72.

Doherty, A., y Forés, A. (2019). Physical activity and cognition: inseparable in the classroom. Frontiers in Education, 4, 1-7.

Duda, J. L., y Ntoumanis, N. (2003). Correlates of achievement goal orientations in physical education. International Journal of Educational Research, 39, 415-436, 


\section{LA MOTIVACIÓN DE LOS ESTUDIANTES UNIVERSITARIOS PARA REALIZAR ACTIVIDAD FÍSICA}

Durán-Vinagre, M. A., Sánchez, S., y Feu, S. (2019). Influencia de la actividad físico- deportiva y la intención de ser físicamente activos en universitarios de la facultad de educación de la UEX. International Journal Developmental Educational Psychology, 5, 83-96.

Escartí, A., Brustad, R. (2002). Estudio de la motivación deportiva desde la perspectiva de la teoría de metas. En: Dosil J, editor. Psicología y rendimiento deportivo. Ourense: GERSAM; 2002. pp. 57-70.

Fernández, E. J., Sáenz-López, P., Almagro, B. J., y Conde, C. (2015). Motivos de práctica de actividad física en universitarios activos. Revista Digital de Educación Física, 6(34), 52-60.

Franco, E., Coteron, J., y Gómez, V. (2017). Relación entre motivación, actividad física realizada en el tiempo libre y la intención futura de práctica de actividad. Estudio comparativo entre adolescentes argentinos y españoles. Revista Euroamericana de Ciencias del Deporte, 6(1), 25-34.

García, W. F. (2019). Sedentarismo en niños y adolescentes: factor de riesgo en aumento. Revista Científica Mundo de la Investigación y el Conocimmiento, 3(1),1602- 1624.

González-Cutre, D., Sicilia, Á., y Fernández, A. (2010). Hacia una mayor comprensión de la motivación en el ejercicio físico: Medición de la regulación integrada en el contexto español. Psicothema, 22, 841-847.

Granero-Gallegos, A., Baena-Extremera, A., Pérez-Quero, F. J., Ortiz-Camacho, M. M., y Bracho-Amador, C. (2012). Analysis of motivational profiles of satisfaction and importance of physical education in high school adolescents. Journal of Sports Science and Medicine, 11, 614-623.

Granero-Gallegos, A., Gómez-López, M., Abraldes, J.A., y Rodríguez-Suárez, N. (2011). Motives of practice in the field of non-competitive physical activity. Espiral Cuadernos del Profesorado, 4(7), 15-22.

Güemes-Hidalgo, M., Ceñal, M. J., e Hidalgo, M. I. (2017). Pubertad y adolescencia. Revista de Formación Continuada de la Sociedad Española de Medicina de la Adolescencia, 5(5), 7-22.

Hagenauer, G., Gläser-Zikuda, M., Moschner, B. (2018). University students' emotions, life-satisfaction and study commitment: A self-determination theoretical perspective. Journal of Further and Higher Education, 42(6), 808-826.

Kim, B. J., y Gill, D. L. (1997). A cross-cultural extensión of goal perspective theory to Korean youth sport. Journal of Sport and Exercise Psychology, 19, 142-155.

Koivula, N. (1999). Sport Participation: differences in motivation and actual participation due to gerder typing. Journal of Sport Behavior, 22(3), 360-380.

Lavie, C. J. Ozemek, C., Carbone, S., Katzmarzyk, P. T., y Blair, S. N. (2019). Sedentary behavior, exercise, and cardiovascular health. Circulation Research, 124(5).

Liu, W., Li, X., Zeng, N., Ayyud, M., Xiong, S., Tao, K., y Pend, Q. (2017). Examining associations among motivation, physical activity and health in chinise college students: a self-determination theory perspective. Journal of Teaching andMedia in Kinesiology, 6, 1-9.

Martínez, H. A., Franco, E., Coterón, J., y Cardona, J. (2015). Relación entremotivación e intención de practicar actividad física. Un estudio entre alumnos colombianos y españoles. Revista Digital de Educación Física, 7(37), 24-33.

Molina-Castillo, J., Castillo, I., y Pablos, C. (2007). Bienestar psicológico y práctica deportiva en universitarios. Motricidad European Journal of Human Movement, 18, 79-91.

Moral, J.E., Martínez, E., y Grao, A. (2013). Sedentarismo, salud e imagen corporal en adolescentes. Sevilla: Wanceulen.

Moreno, J. A., González-Cutre, D., y Ruiz, L. M. (2009). Self-determined motivation and physical education importance. Human Movement, 10(1), 5-11.

Moreno-Murcia, J. A., Cervelló, E., y Martínez, A. (2007). Validación de la Escala de Medida de los Motivos para la Actividad Física-Revisada en españoles: Diferencias por motivos de participación. Anales de psicología, 23(1), 167-176. 
Muyor, J. M., Aguila, C., Sicilia, A., y Orta, A. (2009). Análisis de la motivación autodeterminada en usuarios de centros deportivos. Revista Internacional de Medicina y Ciencias del Deporte, 9, 67-80.

Muyor, J. M., Aguila, C., Sicilia, A., y Orta, A. (2009). Análisis de la motivación autodeterminada en usuarios de centros deportivos. Revista Internacional de Medicina y Ciencias del Deporte, 9, 67-80.

Nie, N. H., Hull, C. H. y Bent, D. H. (2011). Manual del usuario del sistema básico de Ibm Spss Statistics 20. Copyright IBM Corporation.

Nunnally, J. C., y Bernstein, I. H. (1994). Psychometric theory. New York: McGraw-Hill. Pérez, B. M. (2014). Salud: entre la actividad física y el sedentarismo. Anales

Venezolanos de Nutrición, 27(1), 119-128.

Pérez, D., Requena, C., y Zubiaur, M. (2005). Evolución de motivaciones, actitudes y hábitos de los estudiantes de la facultad de ciencias de la actividad física y del deporte de la Universidad de León. Motricidad European Journal of Human Movement, 14, 65-79.

Rippe, J. M. (2019). Lifestyle Medicine 2019: deeper, broader, and more precise.

American. Journal of Lifestyle medicine, 13(5), 436-439.

Ryan, R. M., Frederick, C. M., Lepes, D., Rubio, N., y Sheldon, K. M. (1997). Intrinsic motivation and exercise adherence. International Journal of Sport Psychology, 28(4), 335-354.

Vaquero-Solis, M., Sánchez-Miguel, P. A, Tapia, M. A, Pulido, J. J., y Iglesias Gallego,

D. (2019). Physical Activity as a Regulatory Variable between Adolescents' Motivational Processes and Satisfaction with Life. Int J Environ Res Public Health, 16(15).

Vidarte, J. A., Vélez, C., Sandoval, C., y Alfonso, M. L. (2011). Actividad física: Estrategia promoción de la salud. Hacia la Promoción de la Salud, 16(1), 202-218.

Zamarripa, J. I., Ruiz, F., López, J. M., y Garrido, M. A. (2011). Amotivación de la actividad física en la población mayor de 15 años de Monterrey (México). Retos. Nuevas Tendencias en Educación Física, Deporte y Recreación, 19, 5-9. 
